\section{Scanning laser ophthalmoscope- evoked multifocal ERG (SLO-mfERG) in patients with macular holes and normal individuals}

G Rudolph, P Kalpadakis, M Bechmann,

C Haritoglou and A Kampik

\section{Abstract}

Aims A scanning laser ophthalmoscope (SLO) has been used for multifocal electroretinography (mf ERG) measurements under simultaneous fundus monitoring. The aim of this study was to prove if the SLOmfERG measurement reflects reliably the clinically registered underlying disease, and to demonstrate the importance of its main advantage, fixation monitoring.

Methods In all, 10 patients with macular hole stage II/III were included in the study, and 19 normal individuals served as the control group. The $\mathrm{mf}$ ERG device was combined with an SLO, which was used both as a stimulus and trigger unit as well as a fundusmonitoring system. Monitoring of the fundus was guaranteed by an infrared laser $(780 \mathrm{~nm})$. The stimulus matrix consisted of 61 hexagonal elements, covering $24^{\circ}$ of the posterior pole. We examined both, patients with macular holes and healthy individuals.

Results Compared to normal controls, patients with a macular hole (Gass stage III) showed a significant decrease in response density in the centre of the stimulus array, which correlated well with the morphological alteration observed by clinical examination. However, variation of response density of the central hexagonal area has been proved to be high.

Conclusions SLO-mfERG is a feasible and reliable new technique to investigate macular function under simultaneous fundus control. The main advantage is that control of fixation can be used in order to obtain more reliable results that correlate well with visible fundus abnormalities such as in patients with macular holes. However, further investigations have to be performed in order to overcome sufficiently the problem of fixation instability. Eye (2003) 17, 801-808. doi:10.1038/ sj.eye. 6700502

Keywords: electroretinography; fixation; macular holes; multifocal ERG; scanning laser ophthalmoscope

\section{Introduction}

Generalized diseases of the retina such as, for example, retinitis pigmentosa are detected by standardized Ganzfeld electroretinography (ERG) with high sensitivity, even in early stages of the disease. ${ }^{1-3}$ In localized retinal diseases, for example, in macular diseases, the Ganzfeld ERG is a less useful technique, as its global response is only affected in proportion to the size of the altered retinal area. The pattern ERG (PERG) improved at that point our ability to examine localized retinal diseases. ${ }^{4,5}$ However, attempts to obtain ERG responses from small retinal areas never overcame the main problem of small signal-to-noise ratio. ${ }^{6,7}$ The multifocal ERG allows topographic mapping of retinal function of about $50^{\circ}$ of the central retina. ${ }^{8,9}$ A stimulus array is presented on a monitor and the stimulated areas are changing independently alternating between black and white. The patient has to fixate on a small fixation spot presented in the middle of the stimulation matrix during the whole examination. A major problem of this technique is that no sufficient control of fixation can be performed by the examiner. Therefore, in patients with fixation problems, results may be unreliable, such as in patients with reduced
Eye Clinic, LudwigMaximilians-University Munich, Germany

Correspondence:

G Rudolph

Eye Clinic

Ludwig-Maximilians-

University

Mathildenstrasse 8

D- 80336 München

Germany

Tel.: + 498951603800

Fax: + 498951604569

E-mail: Guenther.Rudolph@ ak-i.med.uni-muenchen.de

Received: 8 July 2002 Accepted in revised form: 18 December 2002

This study has not been supported by any grant or research funding

The authors have no proprietary interest in this study 
compliance, in children or patients with eccentric fixation. To overcome these difficulties, Kondo et al ${ }^{10}$ used an infrared fundus camera combined with a stimulus array of 19 light-emitting diodes (LEDs), allowing the performance of a multifocal ERG under simultaneous fundus control. Seeliger et al ${ }^{11}$ showed the application of scanning laser ophthalmoscope-evoked mfERG (SLO-mfERG) measurements in animals, using a green laser $(515 \mathrm{~nm})$. In many animals, the only way to obtain satisfactory results exists in using a short-wave laser because of the lack of long-wavelength cones. ${ }^{11}$

In this study, we used an SLO-mfERG to examine patients with macular hole (Figure 1) and normal individuals. This new technique offers the possibility to generate a stimulus array with a helium-neon laser under simultaneous control of fixation. ${ }^{12-17}$ The purpose of this study was to assess the feasibility of this method to measure focal retinal function or dysfunction under simultaneous control of fixation.

\section{Materials and methods}

The scanning laser ophthalmoscope (Rodenstock; München, Germany) was used both as a stimulus and trigger device as well as a fundus-monitoring unit. To record an mf ERG, a confocal scanning laser ophthalmoscope is connected to an mfERG device (RETIscan, Roland Consult, Wiesbaden, Germany). The visual stimulus, consisting of a matrix of hexagonal areas flickering concurrently according to a short m-sequence, is displayed directly onto the retina covering a visual

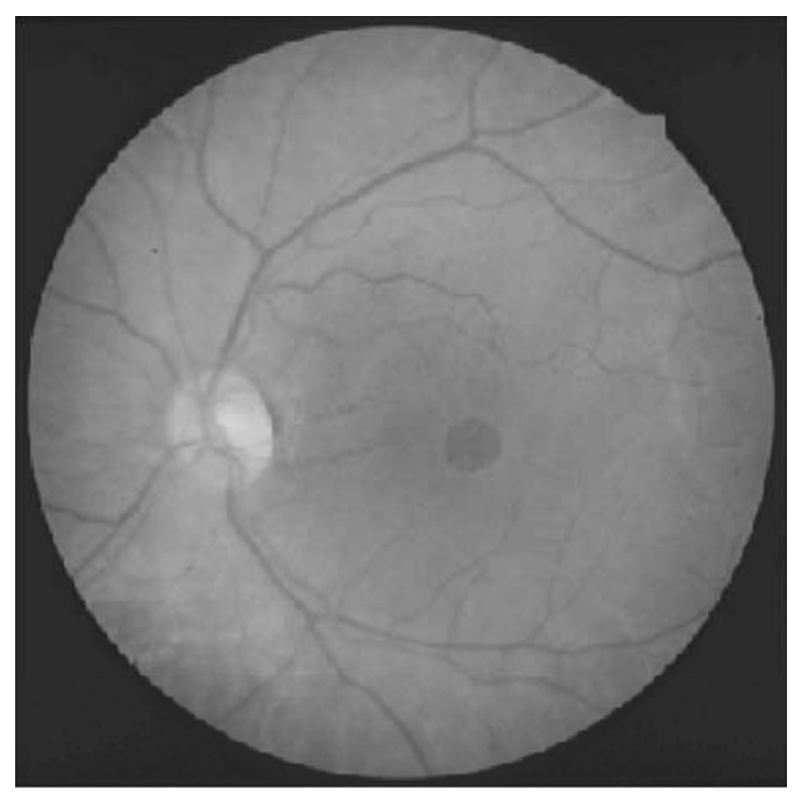

Figure 1 Fundus photography of a patient with a macular hole (Gass II). angle of about $12^{\circ}$ or a diameter of $24^{\circ}$, respectively (Figure 2). ${ }^{18,19}$ It consists of a matrix of 61 hexagonal elements and is generated by a helium-neon laser $(632.8 \mathrm{~nm})$ modulated via an acousto-optic modulator. The matrix has been presented according to a scaling factor 1:4, so that the hexagonal size would be accordingly scaled with eccentricity in order to evoke focal responses of approximately the same amplitude per stimulus element in the response arrays of normal individuals. ${ }^{20}$ With this setting the outermost hexagonal areas were approximately four times larger than the central one. The elements change from red to black or vice versa according to a complete cycle of a binary $\mathrm{m}$ sequence. ${ }^{21}$ The contrast is about $90 \% .^{22}$ The Roland system uses 'short-m-sequences' and a complete measurement cycle consists of 511 steps lasting $40.8 \mathrm{sec}$ $(511 \times 80 \mathrm{~ms})$. Scan time is $20 \mathrm{~ms}$ prohalf-frame ( 50 halfframes/s, 25 frames/s). The examined persons were asked to gaze at a centrally positioned cross. If loss of fixation occurred, the measurement would be interrupted. In cases of unstable fixation the measurement cycle was discarded. The signal of each hexagonal field is calculated by cross-correlation analysis between the $\mathrm{m}$-sequence and the response. The signals are bandpass filtered $(10-100 \mathrm{~Hz})$ and amplified by a two-channel amplifier with a common mode reaction of $>110 \mathrm{~dB}$ and a sensitivity of $1-20 \mu \mathrm{V} /$ div. Simultaneous funduscopy and control of fixation is guaranteed by illumination with an infrared diode laser $(730 \mathrm{~nm})$.

\section{Patients and controls}

In all, 10 patients (ages 46-77 years, mean 64 years) with unilateral diagnosis of an idiopathic macular hole

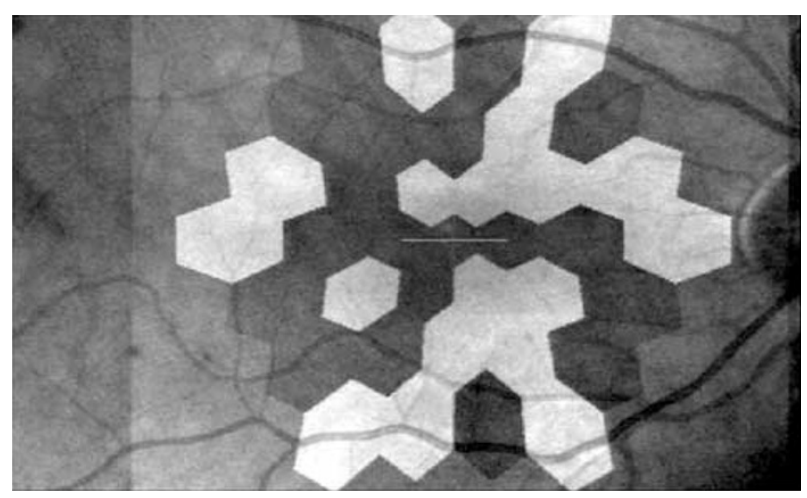

Figure 2 The stimulus pattern on the posterior pole of the eye: the figure displayed was derived from the screen that is used for real-time fundus monitoring, where the fundus picture and the stimulation matrix appear in black and white. Actually, the stimulation pattern generated by the helium-neon laser consists of red light. 
were compared with 19 healthy volunteers (ages 18 to 63 years, mean 40 years) contributing to the study with one eye each. Normal eyes of patients did not serve as controls. Altogether 29 persons were examined with SLO-mfERG using a scaling factor of 1:4. Ophthalmic examination included slit-lamp examination, measurement of visual acuity, funduscopy, and fundus photography. Macular holes were diagnosed by biomicroscopy using a 78 dioptre lens (Volk) and by optical coherence tomography (OCT). Two patients with a macular hole stage II according to Gass showed central fixation, while eight patients with macular hole stage Gass III demonstrated slightly paracentral fixation, but still in the central hexagonal element. Patients with more eccentric fixation were excluded from the study. The visual acuity range was 0.05-0.4.

The recordings were analysed by using concentic ring grouping with evaluation of amplitude density and by displaying the plots. ERGs retrieved from the individual hexagonal areas would also be plotted. Special traces could be selected for detailed data analysis. Amplitude density $\left(\mathrm{nV} / \mathrm{deg}^{2}\right)$ represents the amplitudes for each grouping adjusted for the distance angle of the stimulus element, giving a more accurate view of the actual response amplitudes of each group. ${ }^{23}$ To construct the two and three-dimensional plots the mean of amplitude density was used. ${ }^{9}$

The criteria for the normal controls included in the study were an age of over 18 years, visual acuity of 1.0 with or without correction and contact lens tolerance for both groups. Exclusion criteria for both groups were media opacities, refractive errors of more than 3 dioptres, a history of ocular trauma, diabetic retinopathy, agerelated macular degeneration, or glaucoma. The pupils were not dilated. Refractive errors would be compensated from an integrated system of the SLO. This, generally described, consists of movable mirrors that automatically can be moved, shifting therefore the light beam. Jet contact lens electrodes (Universa SA, La Chaud-de-Fonds, Switzerland) were used. The reference electrode was placed in line with ocular canthi behind the orbital ridge. This research adhered to the tenets of the Declaration of Helsinki and informed consent was obtained from all persons.

\section{Results}

To quantify the electrophysiological results, the responses were grouped by retinal eccentricity in five ring areas. To summarize the results, boxplots showing the 25th and 75th percentile of amplitude density were calculated for both groups of examined persons (healthy individuals and patients with macular holes) (Figure 3).

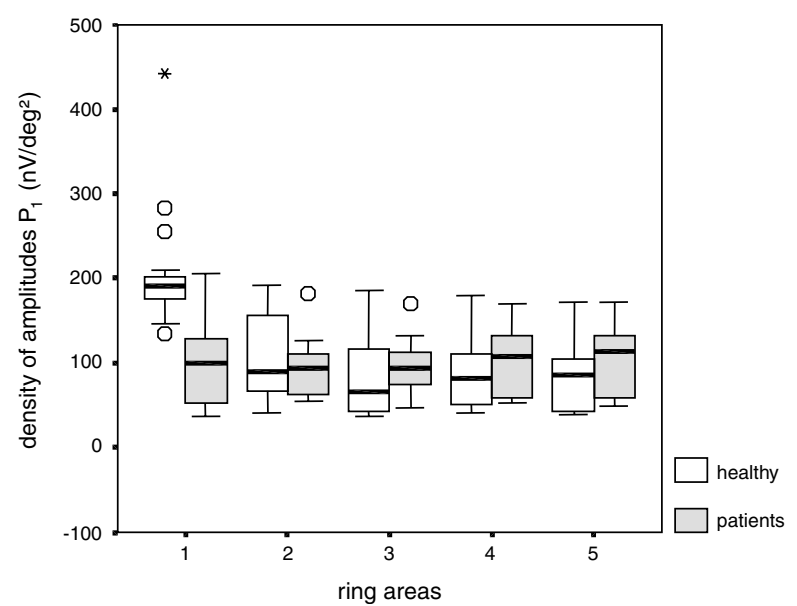

Figure 3 Bold lines in the boxes indicate median, upper and lower edges of the boxes indicate 25th and 75th percentiles accordingly. Upper/lower ends of bars indicate highest/lowest data that is not an outlier. Circles on the graph represent 'outliers'. Outliers are cases with values between 1,5 and 3 box lengths from the upper or lower edge of the box. The box length is the interquartile range. Small stars on the graph represent 'extremes'. The latter are cases with values more than three box lengths from the upper or lower edge of the box.

Fifth, 25th, 75th, 95th percentiles for the normative data and the patients' individual responses can be seen in Table 1. The mean and median values as well as the standard deviation (SD), the interquartile range, and the coefficient of variation of the central hexagonal element and the rings were calculated for each group (Table 2). Our data were tested for and were found inconsistent with the normality assumption (using the Kolmogorov-Smirnov test). The significance level was set at $P=0.05$ and the Mann-Whitney test was applied. Two- and three-dimensional plots of amplitudes P1 both for the healthy individuals as well as for the patients were derived and compared (Figures $4 \mathrm{a}, \mathrm{b}$ and $5 a, b)$.

The amplitudes of the first ring area (central hexagonal element) were found statistically significantly higher in the group of healthy individuals $(P<0.05), \mathrm{M}$ $($ normals $)=198.6, \mathrm{SD}=74.3 ; \mathrm{M}$ (macular holes $)=104.8$, $\mathrm{SD}=54.0$. The amplitudes and latencies of rings $2-5$ areas showed no significant differences (Figures $6 a, b$ and 7a,b, Table 2).

The coefficient of variation (or percentage error) measures the relative scatter of data with respect to the mean and therefore the imprecision in our estimates. It is calculated as 100 (SD)/(mean value of set). In our data set, the coefficient of variation of the response density of the central hexagonal element is relatively higher centrally in the patients with a macular hole as compared to the healthy individuals. 
Table 1 Individual responses of the patients and the 5th, 25th, 75th, and 95th percentiles for the normative data

\begin{tabular}{|c|c|c|c|c|c|}
\hline \multicolumn{6}{|c|}{ Amplitude density (nV/deg2) } \\
\hline Concentic areas & R1 & $R 2$ & $R 3$ & $R 4$ & R5 \\
\hline \multicolumn{6}{|l|}{ Normwerte } \\
\hline 5th Percentile & 62.0 & 42.0 & 37.0 & 42.0 & 39.0 \\
\hline 25th Percentile & 174.0 & 65.0 & 41.0 & 45.0 & 42.0 \\
\hline 75th Percentile & 204.0 & 172.0 & 122.0 & 115.0 & 112.0 \\
\hline 95th Percentile & 283.0 & 192.0 & 151.0 & 160.0 & 168.0 \\
\hline Patient 1 (Gass III) & 52 & 81 & 113 & 123 & 126 \\
\hline Patient 2 (Gass III) & 37 & 60 & 48 & 53 & 49 \\
\hline Patient 3 (Gass III) & 79 & 63 & 86 & 96 & 111 \\
\hline Patient 4 (Gass II) & 167 & 107 & 100 & 135 & 136 \\
\hline Patient 5 (Gass II) & 206 & 182 & 170 & 170 & 172 \\
\hline Patient 6 (Gass III) & 126 & 127 & 132 & 132 & 132 \\
\hline Patient 7 (Gass III) & 88 & 106 & 111 & 108 & 116 \\
\hline Patient 8 (Gass III) & 113 & 110 & 80 & 106 & 105 \\
\hline Patient 9 (Gass III) & 128 & 68 & 75 & 59 & 59 \\
\hline Patient 10 (Gass III) & 52 & 54 & 47 & 52 & 49 \\
\hline
\end{tabular}

Table 2 Amplitude density $\left(\mathrm{nV} / \mathrm{deg}^{2}\right)$ : statistical values of normal individuals and patients with macular holes

\begin{tabular}{|c|c|c|c|c|c|}
\hline & Ring 1 & Ring 2 & Ring 3 & Ring 4 & Ring 5 \\
\hline \multicolumn{6}{|l|}{ Normals } \\
\hline Mean & 198.6 & 109.3 & 86.6 & 90.0 & 86.6 \\
\hline median & 191.0 & 90.0 & 65.0 & 81.0 & 85.0 \\
\hline $\mathrm{SD}$ & 74.3 & 53.0 & 47.3 & 43.8 & 46.6 \\
\hline Coeff.Var. & 37.4 & 48.4 & 54.6 & 48.6 & 53.8 \\
\hline \multicolumn{6}{|l|}{ Macular holes } \\
\hline mean & 104.8 & 95.8 & 96.2 & 103.4 & 105.5 \\
\hline median & 100.5 & 93.5 & 93.0 & 107.0 & 113.5 \\
\hline $\mathrm{SD}$ & 54.0 & 39.3 & 37.7 & 39.2 & 41.0 \\
\hline Coeff. Var. & 51.5 & 41.0 & 39.1 & 37.9 & 38.8 \\
\hline
\end{tabular}

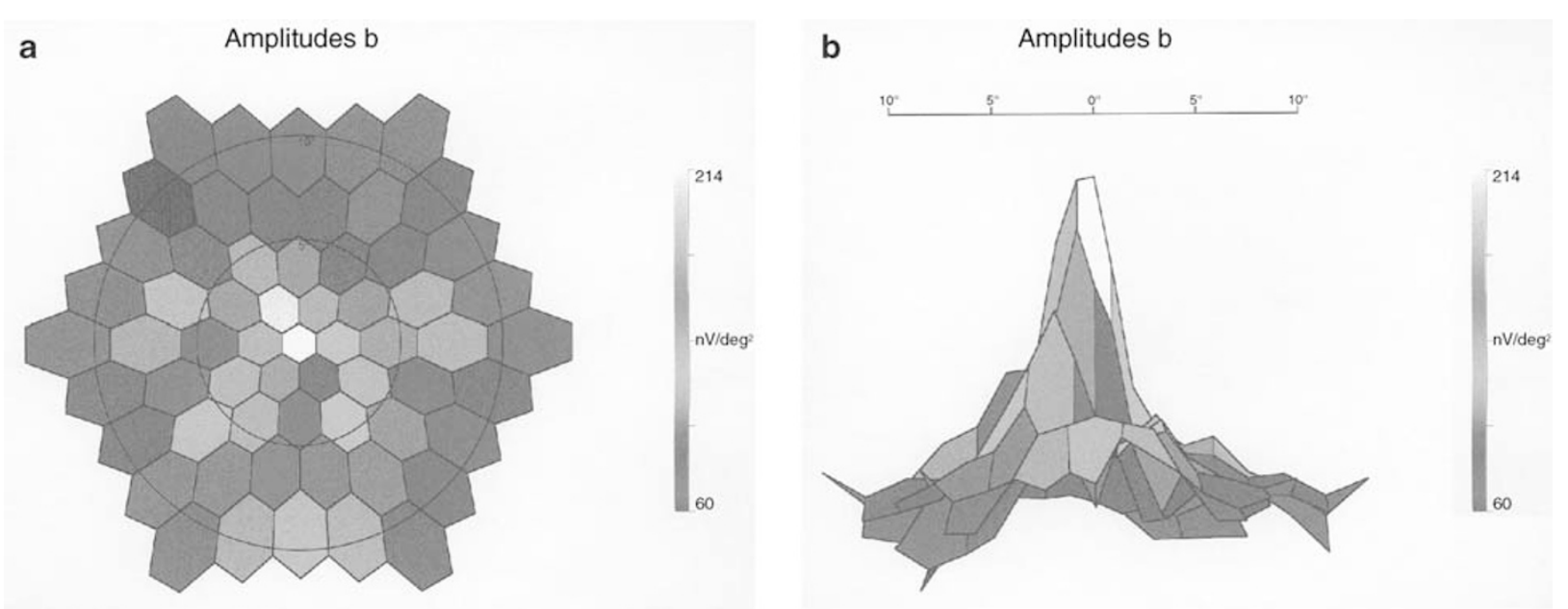

Figure 4 (a) Two-dimensional presentation of amplitudes $P_{1}$ of a healthy person. (b) Three-dimensional presentation (side view) of amplitudes $P_{1}$ of a healthy person showing the central peak. 

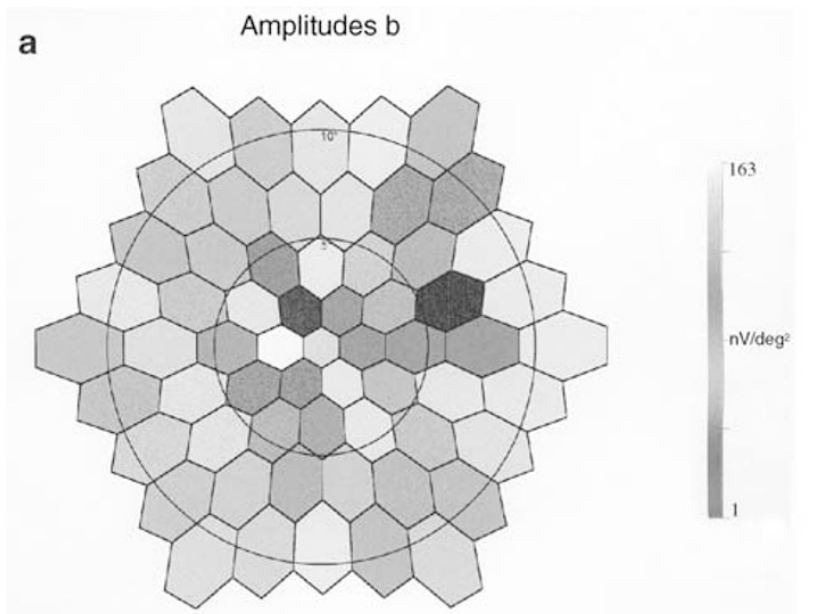

b

Amplitudes b
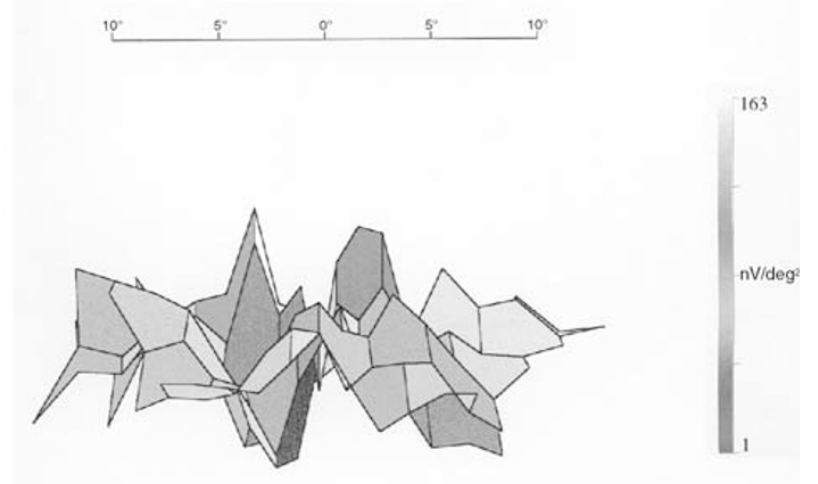

Figure 5 (a) Two-dimensional presentation of amplitudes $P_{1}$ of a patient with macular hole. (b) Three-dimensional presentation (side view) of amplitudes $P_{1}$ of a patient with macular hole showing the reduction of the central peak.

\section{Discussion}

The Ganzfeld ERG produces a generalized response because of the diffuse stimulation of the retina and therefore neglects the effect of limited lesions.

Conventional, monitor stimulated mfERG is a technique capable of measuring retinal function of the posterior pole with high spatial resolution. By multifocal stimulation and by the simultaneous measurement of all the focal responses, a strict correlation of any given signal to a corresponding field exists. ${ }^{24-26}$ By using short

$\mathrm{m}$-sequences, the quality of each measurement cycle can be evaluated separately, and any cycle in which significant artefacts occur can be discarded. The SLOsystem we have employed is also a significant technical advance. It allows the direct projection of the lasergenerated stimulus pattern onto the retina. Moreover, the presence of an infrared diode laser allows simultaneous funduscopy, control of fixation, and imaging of the hexagonal matrix during the whole examination. This is important for all patients with instability of fixation. The importance of an appropriate fixation for a reliable stimulation-response correlation analysis has been lately shown using mfERG recordings and SLOmicroperimetry. ${ }^{27,28}$ Moreover, in a recent study where mfPERG recordings were obtained with an eye tracker from normal individuals, $49 \%$ of the recordings were acquired, while fixation was inadequate. ${ }^{29}$ Loss of fixation would lead to an artificial correlation of the recorded amplitudes to the expected measured retinal areas. The SLO allows continuous monitoring of fixation. The patient can be encouraged and epochs in which control is poor can be discarded. Ultimately, software that compensates for eye movements could be introduced.
Although the main advantage of the mfERG is its spatial resolution, in accordance with Parks et al, ${ }^{30}$ who concluded that repeatability of mfERG can be further enhanced by forming regional groups, concentric rings were grouped around the innermost hexagon of the stimulation array. In concentric macular diseases such as macular holes, an averaging of concentric rings allows a better comparability of responses obtained from the central retina to those derived from more eccentric parts. ${ }^{30}$ As known from the conventional mfERG, response density decreases markedly from the foveal region towards the periphery. This distribution of response density approximately corresponds to the anatomical occurrence of cone distribution of the posterior pole. ${ }^{18-20,31}$ The control group in this study was not age matched, which could have an influence on the results because it is known that an age-related effect exists. Other studies showed a decrease of amplitudes in older patients. ${ }^{32,33}$

In patients with macular hole, a significant reduction of response density could be demonstrated in the centre of the stimulus array. Retinal dysfunction leads to profound amplitude reduction of the central hexagonal element in the SLO-mfERG results of these patients. In the areas corresponding to the elevated margins adjacent to the hole, no significant reduction could be seen in the retinal electrical response. As expected, the strength of the recorded amplitudes of the more peripheral areas does not differ significantly in these patient in comparison with normals. ${ }^{34-38}$

The higher coefficient of variation of amplitudes of the central hexagonal element in patients with macular holes (Table 2) could be explained because of loss of fixation in these patients. However, it raised questions about the 
a

\section{Amplitudes b}

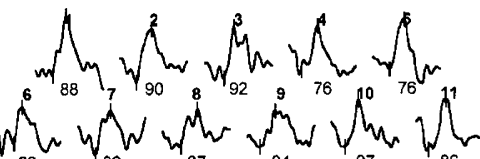

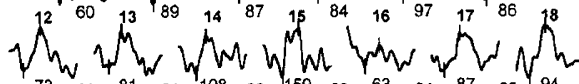

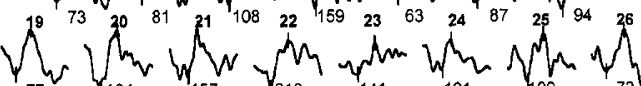

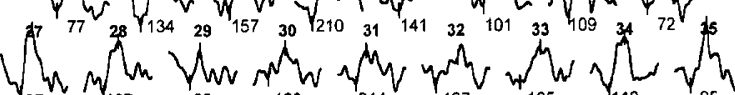

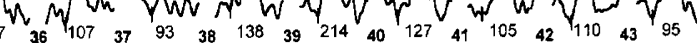

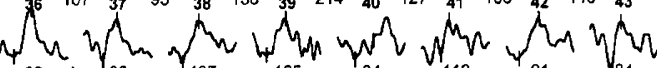

$\begin{array}{llllllllllllll}82 & 44 & 96 & 45 & 137 & 46 & 165 & 47 & 84 & 48 & 112 & 49 & 91 & 90\end{array}$

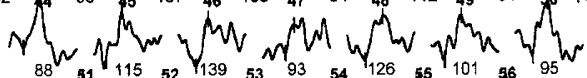

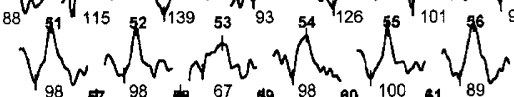

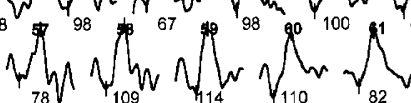

Ampl.b [nV/deg?

b

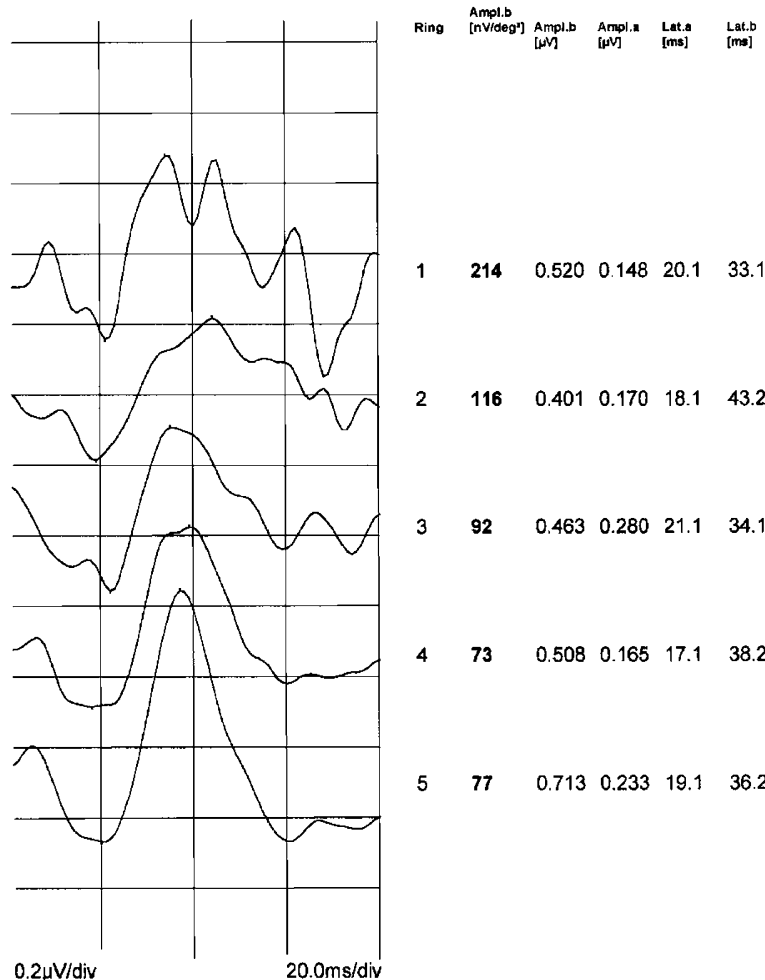

Figure 6 (a) Plots of amplitudes $P_{1}$ from the 61 hexagonal elements of a healthy person. The number of the hexagonal elements is given at the top of the curves while at the bottom appears the amplitude of each field (nV). (b) Concentric ring grouping of a healthy person showing the amplitudes and latencies for each ring. In the first columns is given the amplitude density in $\mathrm{nV} / \mathrm{deg}^{2}$. In the second and third column are the summarized amplitudes $P_{1}$ and $N_{1}$ accordingly per ring grouped area in $\mu \mathrm{V}$. In the fourth and fifth columns are the latencies $P_{1}$ and $N_{1}$ for the summarized curves in $\mathrm{ms}$. measurements' reliability. Fixation loss (through foveal displacement) shifts the central hexagonal element to a more peripheral retinal area, and for a short but variable time period destroys correlation between recorded amplitude and field location. Part of the response attributed to the inner ring will also be generated by the

a

\section{Amplitudes b}

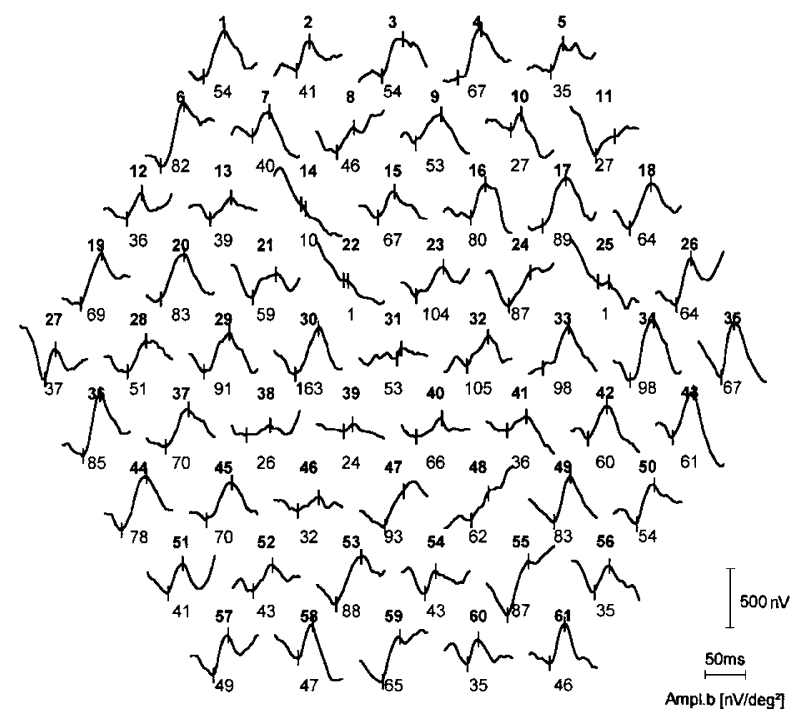

b

\section{Rings}

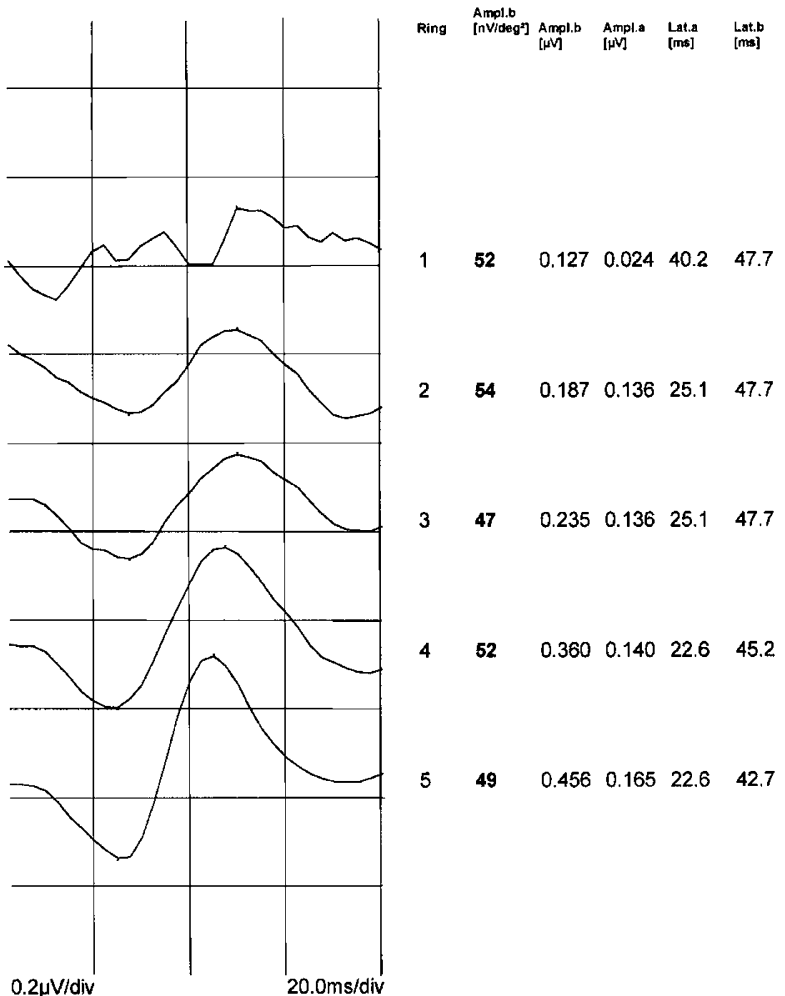


fovea and vice versa, which would be otherwise covered by the central hexagonal element. Our measurements were made under simultaneous fundus visualization and direct fixation control, and still the coefficient of variation implies that such results contain significant errors. Not only in patients but also in healthy individuals, the fixation can pose a real problem. When performing the examination with the monitor version, these possibilities of fixation control are not available. Eyetracking, among other efforts, to solve this problem cannot be a reliable solution, because absence of eye movement does not absolutely mean proper fixation of the eye to the presented target (fixation cross). Thus, when the abovementioned precautions are not available, it is important to remember that scaling may introduce significant difficulties in obtaining reliable data. ${ }^{11,29,39}$

While fixation loss cannot be prevented, the value of our fixation monitoring system depends on the possibility of continuous visualization of fixation during the examination. Fixation depending on concentration, motivation, and retinal function can be, on the one hand, positively affected and on the other objectively evaluated by using the SLO direct fundus control method in order to record mfERGs. In conclusion, it seems that the SLO-mfERG is a useful technique to investigate retinal and in particular macular function under control of fixation, and further studies will be helpful to improve this diagnostic tool.

\section{References}

1 Carr RE, Siegel IM. Fundamentals of Electroretinography. In: Visual Electrodiagnostic Testing. William and Wilkins: Baltimore, London, 1982, pp 3-8.

2 Heckenlively JR, Weleber RG, Arden GB. Testing levels of the visual system: In: Heckenlively JR, Arden GB (eds). Principles and Practice of Clinical Electrophysiology of Vision. Mosby Yearbook. St Louis, 1991, pp 485-493.

3 Marmor M, Zrenner E. Standard for clinical electroretinography (1994 update). Doc Ophthalmol 1995; 89: 199-210.

Figure 7 (a) Plots of amplitudes $P_{1}$ from the 61 hexagonal elements of a patient with macular hole. The number of the hexagonal elements is given at the top of the curves while at the bottom appears the amplitude of each field (nV). No recognizable amplitudes in the fields $14,22,25$, and 48 may be caused by shifts because of microsaccades. (b) Concentric ring grouping of a patient with macular hole showing the amplitudes and latencies for each ring. In the first column is given the amplitude density in $\mathrm{nV} / \mathrm{deg}^{2}$. In the second and third columns are the summarised amplitudes $P_{1}$ and $N_{1}$ accordingly per ring grouped area in $\mu \mathrm{V}$. In the fourth and fifth columns are the latencies $P_{1}$ and $N_{1}$ for the summarised curves in ms.
4 Arden GB, Carter RM, Macfarlan A. Pattern and Ganzfeld electroretinograms in macular disease. Br J Ophthalmol 1984; 68(12): 878-884.

5 Arden GB, Vaegan, Hagg CR. Clinical and experimental evidence that the pattern electroretinogram (PERG) is generated in more proximal retinal layers than the focal electroretinogram (FERG). Ann NY Acad Sci 1982; 388: 580-607.

6 Armington JC, Tepas DI, Kropfl WJ, Hengst WH. Summation of retinal potentials. J Opt Soc Am 1961; 51: 877.

7 Sandberg MA, Ariel M. A hand held two channel stimulator ophthalmoscope. Arch Ophthalmol 1977; 95:1881-1882.

8 Sutter EE, Vaegan. Lateral interaction component and local luminance nonlinearities in the human pattern reversal ERG. Vision Res 1990; 30: 659-671.

9 Sutter EE, Tran D. The field topography of ERG components in man. I. The photopic luminance response. Vision Res 1992; 32: 433-446.

10 Kondo M, Miyake Y, Horiguchi M, Suzuki S, Tanikawa A. Recording multifocal electroretinograms with fundus monitoring. Invest Ophthalmol Vis Sci 1997; 38: 1049-1052.

11 Seeliger M, Narfström K. Functional assesment of the regional distribution of disease in a cat model of hereditary retinal degeneration. Invest Ophthalmol Vis Sci 2000; 41: 1998-2005.

12 Lachenmayr B. Scanning-Laser-Ophthalmoskop (SLO): Prinzip und Wirkungsweise. In: Lund OE, Waubke T (eds) Bücherei des Augenarztes, Vol 133. Stuttgart, Enke Verlag: 1994, pp 54-61.

13 Mainster MA, Timberlake GT, Webb RH, Hughes GW. Scanning laser ophthalmoscopy. Clinical applications. Am Acad Ophthalmol 1982; 89: 853-857.

14 Seeliger MW, Narfström K, Reinhard J, Zrenner E, Sutter E. Continuous monitoring of the stimulated area in multifocal ERG. Doc Ophthalmol 2000; 100: 167-184.

15 Van de Velde FJ, Jalkh AE, Katsumi O, Hirose T, Timberlake G, Scheppens C. Clinical scanning laser ophthalmoscope applications: an overview. In: Nasemann JE, Burk ROW (eds). Scanning laser ophthalmoscopy and tomography, Munich, Quintessenz, 1990, pp 35-47.

16 Webb RH, Hughes GW, Delori FC. Confocal scanning laser ophthalmoscope. Applied Optics 1980; 26: 1492-1492.

17 Woon WH, Fitzke FW, Birg AC, Marshall J. Confocal imaging of the fundus using a scanning laser ophthalmoscope. Br J Ophthalmol 1992; 76: 470-474.

18 Curcio CA, Sloan KR, Kalina RE, Hendrickson AE. Human photoreceptor topography. J Comp Neurol 1990; 292: 497-523.

19 Curcio CA, Sloan KR Jr, Packer O, Hendrickson AE, Kalina RE. Distribution of cones in human and monkey retina. Individual variability and radial asymmetry. Science 1987; 236: $579-582$.

20 Oesterberg GA. Topography of the layer of rods and cones in the human retina. Acta Ophthalmol 1935; 13(6):1-102.

21 Sutter EE. A deterministic approach to non-linear system analysis. In: Printer RB, Nabet B (eds) Nonlinear Vision. CRC Press: Cleveland, 1992, pp 171-220.

22 Teping C, Wolf S, Schippers V, Plesch A, Silny J. Use of the scanning laser ophthalmoscope for recording pattern electroretinography and visual evoked cortical potentials. Klin Mbl Augenheilk 1989; 195: 203-206.

23 Nagatomo A, Nobushima N, Maruiwa F, Arai M, Sawada A. Multifocal electroretinograms in normal subjects. Jpn J Ophthalmol 1998; 42: 129-135. 
24 Kretschmann U, Seeliger M W, Ruether K, Usui T, Apfelstedt-Sylla E, Zrenner E. Multifocal electroretinography in patients with Stargardt's macular dystrophy. Br J Ophthalmol 1998; 82: 267-275.

25 Seeliger M, Kretshmann U, Apfelstedt-Sylla E, Ruther K, Zrenner E. Multifocal electroretinography in retinitis pigmentosa. Am J Ophthalmol 1998; 125: 214-226.

26 Palmowski AM, Sutter EE, Bearse MA, Fung W. Mapping of retinal function in diabetic retinopathy using the multifocal electroretinogram. Invest Ophthalmol Vis Sci 1997; 38: 2586-2596.

27 Wildberger $\mathrm{H}$, Monhart M. Some reflexions concerning the effect of eccentric fixation during $\mathrm{mERG}$ recording. ISCEV XXXIX, Montreal-Mont-Orford, June 2001, p 144 (Abstract).

28 Rohrschneider K, Bueltmann S. Correlation between fundus perimetry and multifocal ERG using the scanning laser ophthalmoscope. Invest Ophthalmol Vis Sci 2001; 42(4): S69 (Abstract).

29 Chisholm JA, Keating D, Parks S, Evans AL. The impact of fixation on the multifocal electroretinogram. Doc Ophthalmol 2001; 102(2): 131-139.

30 Parks S, Keating D, Williamson TH, Evans AL, Elliot AT, Jay JL. Functional imaging of the retina using the multifocal electroretinograph: a control study. Br J Ophthalmol 1996; 80: 831-834.

31 Rudolph G, Kalpadakis P, Bechmann M, La Rocca G, Hormann C, Berninger T. Scanning laser ophthalmoscopeevoked multifocal-ERG ((SLO-m-ERG) by using short m-sequences. Eur J Ophthalmol 2002; 12(2): 109-116.

32 Jackson GR, Ortega J, Girkin C, Rosenstiel CE, Owsley C. Aging-related changes in the multifocal electroretinogram. J Opt Soc Am A Opt Image Sci Vis 2002; 19: $185-189$.

33 Nabeshima T. The effect of ageing on the multifocal electroretinogramm. Jpn J Ophthalmol 2001; 45: 114-115.

34 Barber $\mathrm{C}$. The multifocal technique: a new era in clinical electrophysiology of vision. Focus 2002; 21.

35 Haritoglou C, Gass C, Schaumberger M, Ehrt O, Gandorfer A, Kampik A. Macular changes after peeling of the internal limiting membrane in macular hole surgery. $A m \mathrm{~J}$ Ophthalmol 2001; 132: 363-368.

36 Moschos M, Apostolopoulos M, Ladas J, Theodossiadis P, Malias J, Moschou $\mathrm{M}$ et al. Multifocal ERG changes before and after macular hole surgery. Doc Ophthalmol 2001; 102(1): 31-40.

37 Si YJ, Kishi S, Aoyagi K. Assessment of macular function by multifocal electroretinogram before and after macular hole surgery. Br J Ophthalmol 1999; 83(4): 420-424.

38 Terasaki H, Miyake Y, Nomura R, Piao CH, Hori K, Niwa T et al. Focal macular ERGs in eyes after removal of macular ILM during macular hole surgery. Invest Ophthalmol Vis Sci 2001; 42: 229-234.

39 Rohrschneider K, Bueltmann S. Correlation between fundus perimetry and multifocal ERG using the scanning laser ophthalmoscope. Invest Ophthalmol Vis Sci 2001; 42(4): 69 (Abstract). 